Artículo de investigación e innovación / Reflexión

\title{
Indicadores de carga del cuidado familiar de personas con enfermedad crónica: relevantes en salud pública
}

\author{
Cost Indicators of Family Care of People with Chronic \\ Disease: Relevant in Public Health

\section{Os indicadores de maior peso no cuidado familiar de doentes crônicos: são relevantes na saúde pública}

Lucy Barrera-Ortiz, Enf., Mg. *

Sonia Patricia Carreño, Enf., Mg., PhD. **

Gloria Mabel Carrillo, Enf., Mg., PhD. (C) ***

Lorena Chaparro-Díaz, Enf., PhD. ****

\section{Resumen}

Introducción: El abordaje de la enfermedad crónica implica contar con un esquema de mediciones objetivas, pertinentes, precisas, confiables y costo efectivas. Objetivo. Establecer el sistema de indicadores requeridos para medir la carga familiar del cuidado de personas con enfermedad crónica. Metodología. Reflexión derivada de investigación a partir del "Modelo para la Disminución de la Carga del Cuidado de la Enfermedad Crónica" (MDCC-EC) y con base en la Guía Universidad Nacional de Colombia Sistema de Mejor Gestión (UN SIMEGE), en la que se construyen y formulan los indicadores para la medición y seguimiento de los procesos tendientes a disminuir la carga familiar del cuidado de la enfermedad crónica. Resultados: La metodología planteada en la Guía UN SIMEGE permitió presentar indicadores para medir cinco características asociadas con la carga familiar del cuidado de la enfermedad crónica que están descritas como parte

\author{
Olga Janeth Gómez, Enf., Mg PhD. ***** \\ Beatriz Sánchez-Herrera, Enf., MSN. ****** \\ Elizabeth Vargas-Rosero, Enf., Mg. *******
}

\begin{abstract}
fundamental del MDCC-EC. Estas incluyen el acceso, la continuidad, la seguridad, el fortalecimiento de la propia capacidad y el soporte social con el uso de las tecnologías de información y comunicación (TIC) Conclusiones: Los 19 indicadores establecidos permiten en conjunto medir la carga familiar del cuidado de la enfermedad crónica. Esta medición es necesaria e indispensable para conocer, entender, orientar y corregir la ejecución de las estrategias, acciones y resultados de la aplicación del MDCC-EC en lo referente a las personas con enfermedad crónica y sus familias. Comunicar los resultados de la consecución de las metas para saber cómo se plasman los planteamientos teórico conceptuales del MDCC-EC, dando cumplimiento a los requisitos de los usuarios con el servicio permite, de una parte, evaluar y de otra, continuar agregando valor al sistema de salud. [Barrera-Ortiz L, Carreño SP, Carrillo GM, Chaparro-Díaz L, Gómez OJ, Sánchez-Herrera B, VargasRosero E. Indicadores de carga del cuidado familiar de personas con enfermedad crónica: relevantes en salud pública. MedUNAB 2015; 18 (2): 125-134]
\end{abstract}

* Enfermera, Magister en Enfermería, Profesora Titular Pensionada, Facultad de Enfermeria, Universidad Nacional de Colombia, Bogotá-Colombia.

** Enfermera, Magister en Enfermería, Doctora en Enfermería (C); Profesora Auxiliar, Facultad de Enfermería, Universidad Nacional de Colombia, Bogotá-

Colombia.

*** Enfermera, Magister en Enfermería, Doctora en Enfermería (C), Profesora Asociada, Facultad de Enfermería, Universidad Nacional de Colombia, BogotáColombia.

**** Enfermera, Doctora en Enfermería, Profesora Asociada, Facultad de Enfermería, Universidad Nacional de Colombia, Bogotá-Colombia.

***** Enfermera, Magister en Enfermería, Doctora en Enfermería, Profesora Asociada, Facultad de Enfermería, Universidad Nacional de Colombia, BogotáColombia.

***** Enfermera, Master in Science of Nursing, Profesora Titular Pensionada, Facultad de Enfermería, Universidad Nacional de Colombia, Bogotá-Colombia.

******Enfermera, Magister en Enfermería, ProfesoraAsociada, Facultad de Enfermería, Universidad Nacional de Colombia, Bogotá-Colombia.

Correspondencia: Gloria Mabel Carrillo, Profesora Asociada, Universidad Nacional de Colombia. Facultad de Enfermería. Calle 44 N 45-67, Unidad Camilo Torres, Bloque B2, Oficina 804. Correo electrónico: gmcarrillog@unal.edu.co. 
Palabras Clave: Indicadores de Calidad de la Atención de Salud; Indicadores; Enfermedad Crónica; Costo de Enfermedad; Cuidadores.

\section{Abstract}

Introduction: Addressing chronic illness implies having an outline of objective, pertinent, accurate, reliable, and costeffective measures. Objective: This paper's objective is to establish a system of indicators required to measure the relative cost of family care of people with chronic disease. Metodology: Starting with the "Model for the Reduction of Care Burden of Chronic Disease" MDCC- EC, and based on the UN SIMEGE Guide, for the development of indicators in order to measure and monitor the processes aimed at reducing the cost of family care of chronic disease. Results: The methodology proposed in the UN SIMEGE Guide allowed the construction of indicators for five characteristics associated with family care for people with chronic disease. These characteristics are described as a fundamental component of the MDCC-EC and they include:The care access, care continuity, care safety, the self-care capacity and the social support with the use of Information and Communication Technologies (ICTs). Conclusions: The 19 indicators established allow the measurement of the cost of family care for people with chronic disease. This measurement is necessary and indispensable to know, understand, guide and correct implementation of strategies, actions and results of the implementation of MDCC-EC in relation to ill people and their families. These indicators are essential in order to communicate the results of the fulfillment of the goals for how the conceptual theoretical approaches of the MDCC-EC are met in compliance with the requirements of users of services and to continue improving the given services adding value to them. [Barrera-Ortiz L, Carreño SP, Carrillo GM, ChaparroDíaz L, Gómez OJ, Sánchez-Herrera B, Vargas-Rosero E. Cost indicators of family care of people with chronic disease: relevant in public health. MedUNAB 2015; 18 (2): 125-134]

Keywords: Quality Indicators; Health Care; Indicators; Chronic Disease; Cost of IIIness; Caregivers.

\section{Introducción}

La enfermedad crónica (EC) es un trastorno funcional (orgánico y mental), que dura más de 6 meses, exige modificar el estilo de vida; es permanente, multicausal, genera incapacidad residual, requiere largos periodos de cuidado, tratamiento especializado y control, e implica a la persona, a su familia y a su contexto(1).

Desde el punto de vista epidemiológico la EC es causa del $63 \%$ de muertes en el mundo, en donde las dolencias cardiovasculares son el $48 \%$ de estas muertes, el cáncer de $2 \%$, la enfermedad respiratoria crónica del $12 \%$ y la diabetes del $3.5 \%$. La tendencia de la EC va en aumento con 36 millones de personas que murieron por esta causa en 2008 y una proyección de 55 millones para 2030, con $80 \%$ de esas muertes en los países de ingreso bajo y medio(2).

\section{Resumo}

Introdução: $\mathrm{Na}$ abordagem da doença crônica é necesario utilizar o esquema de medidas objetivas, relevantes, precisas, confiáveis e custo efetivo. Objetivo. Estabelecer o sistema de indicadores necessários para medir o peso familiar relacinado com o cuidado das pessoas com doenças crônicas. Metodologia. A reflexão é decorrente do "Modelo de Custo Reduzido Chronic Disease Cuidado" (MDCC- CE) além de ter como base a Guia da Universidade Nacional da Colômbia Melhor Sistema de Gestão (SIMEGE ONU), na qual são construídos os indicadores para medir e observar os processos que visam a redução dos encargos famíliares no cuidado da doença crónica. Resultados: Ametodologia proposta na Guia Um SIMEGE possibilitou mostrar indicadores para medir cinco características que permitem medir a sobrecarga familiar de cuidados para doenças crônicas que são descritos como uma parte fundamental do MDCC-CE. Estes incluem o acesso, continuidade, segurança, capacitação e apoio social em si com o uso de tecnologias de informação e comunicação (TIC). Conclusões: Os 19 indicadores estabelecidos permitem medir a carga familiar nos cuidados da doença crónica. Esta medida é necessária e indispensável para conhecer, compreender, orientar e corrigir a implementação de estratégias, ações e resultados na pratica do MDCC-CE em relação às pessoas com doenças crônicas e suas famílias. Comunicar os resultados da aquisição das metas permite conhecer como as abordagens teóricas conceituais do MDCC-CE responde às exigências dos usuários com o serviço, permitindo tanto a avaliação como adicionar valor ao sistema de saúde. [Barrera-Ortiz $L$, Carreño SP, Carrillo GM, Chaparro-Díaz L, Gómez OJ, Sánchez-Herrera B, Vargas-Rosero E. Os indicadores de maior peso no cuidado familiar de doentes crônicos: são relevantes na saúde pública. MedUNAB 2015; 18 (2): 125134]

Palavras-chave: Indicadores de Qualidade em Assistência à Saúde; Indicadores; Doença Crônica; Efeitos Psicossociais da Doença; Cuidadores.

En 2012 la Asamblea General de la Organización de las Naciones Unidas convocó a la revisión del problema de la EC bajo el argumento de que esta representa uno de los principales problemas del siglo XXI que contribuye a la pobreza y solicitó acciones de los diferentes países en términos de mejorar las capacidades para reducir riesgos y para fortalecer los sistemas de salud con mayor seguimiento y evaluación; además, solicitó a la Organización Mundial de la Salud crear un marco mundial de vigilancia general con recomendaciones para establecerlo. En el marco de la vigilancia sobre la EC en el mundo, la OMS señala que en la proporción de mortalidad en Colombia en 2014 incluidas todas las edades y ambos sexos se asoció con EC en un $71 \%$ de los casos(3).

Al revisar el sistema de salud colombiano se encuentra que este no está suficientemente preparado para asumir el reto de la EC. De una parte, trata igual a las personas cuando padecen enfermedad aguda o EC, sus profesionales no tienen la 
formación requerida para asumir este reto y en consecuencia se genera insatisfacción de las necesidades de los pacientes y sus familiares, un deterioro prematuro e institucionalización innecesaria que termina implicando un mayor costo. Puede señalarse que bajo criterios internacionales el sistema de salud es un sistema inmaduro (4). El incremento exponencial de la EC y la baja preparación del sistema son parte de las razones para haberla declarado la mayor prioridad de la investigación en salud en Colombia (5).

Los abordajes conceptuales propuestos para atender a las personas han ido cambiando de los biomédicos conocidos como "curativos" a los de cuidado, que buscan la integralidad, se centran en la persona que tiene una enfermedad, buscan atender el significado de estar enfermo, consideran la importancia del contexto, integran a la familia como parte de la experiencia e identifican en ella al cuidador principal, buscan aliviar el sufrimiento y hacer más fácil la experiencia (6). Estos abordajes se centran en la experiencia de la salud para fortalecer la autonomía y dignificar al ser humano.

A partir de los abordajes de cuidado se ha encontrado que la EC genera impacto en el enfermo y en su contexto, especialmente en su familia quienes cuidan la mayor parte del tiempo a las personas enfermas (7-8). En América Latina se ha reportado percepción de carga familiar asociada con el cuidado de las personas con enfermedad crónica $(1,9)$ y a nivel mundial se ha señalado cómo estas familias ingresan en círculos de enfermedad y pobreza (10). Es decir, desde la perspectiva económica la familia también se ve afectada por la falta de acceso real a la seguridad social y atención en salud (11-13); por factores asociados al ingreso o distribución de sus recursos cuando se atiende a una persona enferma (1418); por aspectos de política macroeconómica que buscan de manera infructuosa evitar fenómenos de pobreza para quienes enfrentan esta situación (19-21); por el área donde viven, con mayor impacto en zonas rurales (22-24) y por las condiciones de gasto asociado con dolencias específicas(2529). Se suman a estas situaciones las reconocidas inequidades en salud entre regiones, niveles educativos, $\mathrm{y}$ estratos sociales, entre otros $(30,31)$. Si bien en América Latina se han adelantado diferentes estudios de carga de la EC donde se valoran los años de vida saludable perdidos
(AVISAS) o años de vida ajustados por discapacidad (AVAD) (32), estos muestran un panorama regional del impacto económico de la EC con base en el cual se orientan los recursos destinados a la salud y se comparan resultados de los esfuerzos de cada sistema de seguridad social (33). Sin embargo, las medidas de carga disponibles han sido generadas con base en el costo social y económico por paciente. Estas medidas deben ser más amplias para considerar otros determinantes y factores asociados con el cuidado de la salud (34).

Por otro lado, además de abordar la EC desde sus etapas $(35,36)$, se requiere una perspectiva que incluya los problemas que afectan a las personas con EC y a sus familias tales como a) La modificación del estilo de vida para mejorar el pronóstico; b) El alivio del dolor, la limitación y la mutilación; c) La alteración de la capacidad de socializar y d) El afrontamiento de una gran pérdida o la muerte (1). La carga del cuidado incluye la mirada de quien cuida la experiencia de la salud humana. Se entiende por esta "Carga del cuidado" el esfuerzo adicional y desgastante que es requerido para satisfacer las necesidades de las personas con enfermedad crónica, de los mismos pacientes, sus cuidadores familiares, los profesionales de la salud, las instituciones, el sistema de salud y la sociedad en general. El constructo "carga del cuidado de la EC" representa un fenómeno no tangible, que puede ser inferible mediante la percepción, la conducta o el impacto que esta genera (37). Los modelos en el campo de la salud responden a las diferentes problemáticas estudiadas y en algunas ocasiones se requiere más de un modelo para abordar estos fenómenos (38). En el mundo se ha abordado el problema de la carga del cuidado mediante modelos de atención asociados con la EC tales como los explicativos de la EC, los que están orientados al manejo de EC específicas, y los basados en el Cuidado en situaciones de EC. El Modelo de Cuidado Crónico (Chronic Care Model) de la OMS es quizá uno de los más reconocidos en el campo (39). Con el propósito de orientar la práctica e investigación en el campo, se planteó el Modelo para la disminución de la Carga del Cuidado de la Enfermedad Crónica (MDCC-EC) que fue validado por 36 expertos nacionales e internacionales como un paso previo a su aprobación. La figura 1 esquematiza el modelo generado.

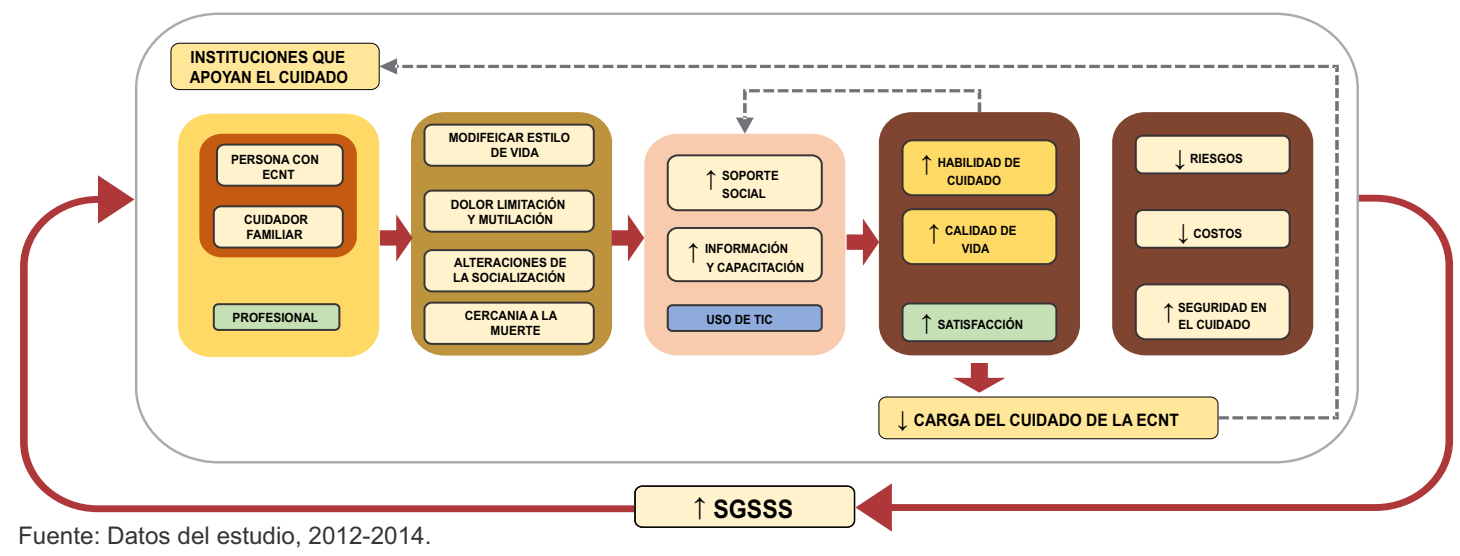

Figura 1. Modelo Para la Disminución de La Carga del Cuidado de Personas con Enfermedad Crónica. 
Para la operacionalización del MDCC-EC fue necesario conocer la evolución de cinco aspectos específicos relacionados con el cuidado de la salud de las personas con enfermedad crónica y sus familias: el acceso, la continuidad, la seguridad, el fortalecimiento de la capacidad de cuidar y el soporte social con empleo de TIC disponibles. Esta pretensión exigió determinar unos indicadores y establecer la información requerida en forma de datos para posibilitar su medición.

Se entiende como indicador la expresión cualitativa o cuantitativa observable que permite describir características, comportamientos o fenómenos de la realidad a través de la evolución de una variable o el establecimiento de una relación entre estas (40).

Medir los procesos que componen el modelo es útil para conocer, entender, orientar y corregir la ejecución de las estrategias, acciones y resultados del mismo. Permite comunicar los resultados del cumplimiento de las metas de manera que se establezca con certeza el nivel de logros específicos de cada una de las estrategias proyectadas con base en el modelo y en caso de que estos no sean los esperados poder determinar las causas y establecer las acciones más pertinentes para corregir las fallas y evitar que vuelvan a ocurrir. Las mediciones que se plantean deben ser transparentes, entendibles, objetivas, pertinentes, precisas, oportunas, confiables y costo efectivas (40). En este caso los indicadores materializan lo que se quiere observar y medir, y se consideran el principal parámetro para el control de la gestión para lo cual deben ser simples, medibles y comunicables (40).

De acuerdo con lo descrito, el objetivo del presente artículo fue establecer el sistema de indicadores requeridos para medir la carga familiar del cuidado de personas con enfermedad crónica.

\section{Metodología}

Reflexión derivada de investigación que hace parte del Programa para la Disminución de la Carga de la EC en Colombia. Tuvo en cuenta criterios éticos dispuestos en la Resolución 008430 de 1993(41) y las pautas éticas internacionales para la investigación biomédica con seres humanos (CIOMS) (42) y contó con la aprobación de los Comités de ética institucionales.

A partir del "Modelo para la Disminución de la Carga del Cuidado de la Enfermedad Crónica" MDCC- EC" y con base en la Guía Universidad Nacional de Colombia Sistema de Mejor Gestión (UN SIMEGE) (40), se construyen y formulan los indicadores para la medición y seguimiento de los procesos correspondientes a las personas con EC y sus CF en la búsqueda de disminución de la carga familiar del cuidado de la enfermedad crónica. Para ello se cumplieron los siguientes pasos: 1) Determinar proceso y objetivos de cada componente; 2) Determinar las variables a medir; 3) Identificar la fuente de los datos; 4) Definir la fórmula de cálculo; 5) Establecer la meta y los rangos; 6)Definir periodicidad de recopilación de los datos de la variable; 7) Determinar registro de recopilación; 8) Definir responsable de la medición; 9) Revisar y aprobar indicador; 10) Documentar la Hoja de Vida del Indicador; 11) Escoger herramienta estadística de calidad; 12) Definir responsable del análisis y evaluación de datos; 13) Alimentar datos y resultados del indicador; 14) Evaluar y analizar resultados; 15) Tomar acciones de mejora y 16) Presentar y publicar resultados.

\section{Resultados}

A partir de la aplicación de la metodología se definieron 19 indicadores que en conjunto permiten ver si se ha disminuido la carga del cuidado de la EC a nivel familiar. En la tabla 1 se presenta cada uno de los indicadores a partir de su hoja de vida. En la tabla 2 se ilustra a manera de ejemplo la hoja de vida del indicador de acceso al cuidado que aborda la carga financiera familiar.

\section{Discusión}

El uso de indicadores como una herramienta fundamental de la gestión en salud ha sido ampliamente estudiado por servicios (43), como una forma de revisión de la realidad de vida urbana (44), en el desempeño profesional de la enfermería (45) y en la investigación (46), entre otros. En todos los casos la literatura señala que a partir de los indicadores se puede lograr observar y medir el fenómeno a evaluar.

Sin embargo, tal como ha sido señalado aunque todo es susceptible de ser medido, el asunto radica en cómo y con qué medirlo. Esto implica metodologías confiables, instrumentos claros y precisos que den resultados significativos y que puedan incidir frente a las políticas en salud (47).

Los indicadores son un aporte complementario a las mediciones de la carga de la enfermedad existentes y que han sido aplicadas en el contexto de América Latina (32). Estos responden a los llamados de tener unas miradas más amplias para considerar otros determinantes (34) tales como los factores asociados con el cuidado de la salud.

Los indicadores reportados han mirado la situación de enfermedad crónica bajo paradigmas de cuidado y por ello responden a la experiencia de la salud humana en este caso (35), ellos permiten medir estrategias aplicadas frente a los principales problemas del cuidado en medio de la cotidianidad(1). 
Tabla 1. Indicadores propuestos para medir la carga del cuidado familiar de una persona con enfermedad crónica (EC).

\section{PROCESO: DISMINUIR LA CARGA DEL CUIDADO DE PERSONAS CON ENFERMEDAD CRÓNICA Y SUS FAMILIAS}

\begin{tabular}{|c|c|c|c|c|}
\hline Estrategia & Objetivo & $\begin{array}{c}\text { Persona con } \\
\text { enfermedad crónica }\end{array}$ & $\begin{array}{l}\text { Cuidador } \\
\text { familiar }\end{array}$ & $\begin{array}{l}\text { Diada Cuidador } \\
\text { Familiar- Receptor } \\
\text { del Cuidado }\end{array}$ \\
\hline $\begin{array}{l}\text { Acceso al } \\
\text { cuidado }\end{array}$ & $\begin{array}{l}\text { Mejorar el acceso } \\
\text { al cuidado de la } \\
\text { persona con } \\
\text { enfermedad } \\
\text { crónica y de su } \\
\text { cuidador familiar. }\end{array}$ & $\begin{array}{l}\text { 1. ( } \mathrm{N}^{\circ} \text { de personas con } \mathrm{EC} \\
\text { que perciben ser menor } \\
\text { carga / } \mathrm{N}^{\circ} \text { de personas con } \\
\mathrm{EC} \text { que participan en el } \\
\text { programa disminución de la } \\
\text { carga del cuidado) } \times 100 \text {. }\end{array}$ & $\begin{array}{l}\text { 2. ( } \mathrm{N}^{\circ} \text { de cuidadores } \\
\text { familiares que perciben } \\
\text { menor sobrecarga con } \\
\text { el cuidado (medida con } \\
\text { Encuesta de Zarit) / } \mathrm{N}^{\circ} \\
\text { de cuidadores familiares } \\
\text { de personas con EC } \\
\text { que participan en el } \\
\text { programa disminución } \\
\text { de la carga del cuidado) } \\
\text { x } 100 \text {. }\end{array}$ & $\begin{array}{l}\text { 3. }\left(\mathrm{N}^{\circ} \text { de familias que }\right. \\
\text { logran mantener } 0 \\
\text { disminuir la carga } \\
\text { financiera con el } \\
\text { cuidado de la persona } \\
\text { enferma / } \mathrm{N}^{\circ} \text { de } \\
\text { familias que son } \\
\text { incluidas en el } \\
\text { programa de } \\
\text { disminución de la } \\
\text { carga financiera con el } \\
\text { cuidado) x } 100 \text {. }\end{array}$ \\
\hline $\begin{array}{l}\text { Continuidad del } \\
\text { cuidado }\end{array}$ & $\begin{array}{l}\text { Incremento de la } \\
\text { competencia de } \\
\text { cuidar en el hogar. } \\
\text { Si el paciente es } \\
\text { independiente y } \\
\text { está cognitivamente } \\
\text { intacto será medida } \\
\text { en el paciente. Si } \\
\text { es dependiente o } \\
\text { tiene su capacidad } \\
\text { cognitiva afectada, } \\
\text { será medida en el } \\
\text { cuidador familiar }\end{array}$ & \multicolumn{3}{|c|}{$\begin{array}{l}\text { 4. ( } \mathrm{N}^{\circ} \text { de pacientes o cuidadores familiares que mejoran su nivel de competencia } \\
\text { de cuidado en el hogar/ } \mathrm{N}^{\circ} \text { de pacientes o cuidadores que son incluidos en el } \\
\text { programa de plan de egreso hospitalario) } \times 100 .\end{array}$} \\
\hline \multirow[t]{2}{*}{$\begin{array}{l}\text { Seguridad del } \\
\text { cuidado }\end{array}$} & \multirow{2}{*}{$\begin{array}{l}\text { Para el caso del } \\
\text { paciente, mejorar } \\
\text { el conocimiento, } \\
\text { clarificar las pautas } \\
\text { de prevención, } \\
\text { mejorar la actitud } \\
\text { personal frente al } \\
\text { riesgo. Disminuir el } \\
\text { riesgo materializado } \\
\text { y fortalecer la } \\
\text { percepción global } \\
\text { de seguridad. } \\
\text { Para el caso del } \\
\text { cuidador familiar, } \\
\text { mejorar conocimiento, } \\
\text { clarificar las pautas } \\
\text { de prevención, }\end{array}$} & $\begin{array}{l}\text { 5. ( } \mathrm{N}^{\circ} \text { de pacientes que } \\
\text { mejoran el nivel de } \\
\text { conocimiento del riesgo } \\
\text { institucional } / \mathrm{N}^{\circ} \text { de } \\
\text { pacientes que son incluidos } \\
\text { en el programa de cuidado } \\
\text { seguro) x } 100 \text {. }\end{array}$ & $\begin{array}{c}\text { 6. ( } \mathrm{N}^{\circ} \text { de cuidadores } \\
\text { familiares que mejoran } \\
\text { el nivel de conocimiento } \\
\text { del riesgo institucional / } \\
\mathrm{N}^{\circ} \text { de cuidadores } \\
\text { familiares que son } \\
\text { incluidos en el programa } \\
\text { de cuidado seguro) x } 100 \text {. }\end{array}$ & $\begin{array}{l}\text { 7. ( } \mathrm{N}^{\circ} \text { de pacientes que } \\
\text { presentaron eventos } \\
\text { adversos/ } \mathrm{N}^{\circ} \text { de pacientes } \\
\text { hospitalizados con los } \\
\text { que se trabajaron } \\
\text { estrategias mejorar su } \\
\text { percepción sobre las } \\
\text { condiciones de } \\
\text { seguridad } \times 100 \text {. }\end{array}$ \\
\hline & & $\begin{array}{l}\text { 8. }\left(\mathrm{N}^{\circ} \text { de pacientes que }\right. \\
\text { clarifican las pautas de } \\
\text { prevención del riesgo } \\
\text { institucional / } \mathrm{N}^{\circ} \text { de } \\
\text { pacientes que son incluidos } \\
\text { en el programa de cuidado } \\
\text { seguro) x } 100 \text {. }\end{array}$ & $\begin{array}{c}\text { 9. }\left(\mathrm{N}^{\circ} \text { de cuidadores }\right. \\
\text { familiares que mejoran las } \\
\text { pautas de prevención del } \\
\text { riesgo institucional } / \mathrm{N}^{\circ} \text { de } \\
\text { cuidadores familiares } \\
\text { que son incluidos en el } \\
\text { programa de cuidado } \\
\text { seguro }) \times 100 \text {. }\end{array}$ & \\
\hline
\end{tabular}




\begin{tabular}{|c|c|c|c|c|}
\hline Estrategia & Objetivo & $\begin{array}{c}\text { Persona con } \\
\text { enfermedad crónica }\end{array}$ & $\begin{array}{l}\text { Cuidador } \\
\text { familiar }\end{array}$ & $\begin{array}{l}\text { Diada Cuidador } \\
\text { Familiar- Receptor } \\
\text { del Cuidado }\end{array}$ \\
\hline \multirow[t]{3}{*}{$\begin{array}{l}\text { Seguridad del } \\
\text { cuidado }\end{array}$} & \multirow[t]{3}{*}{$\begin{array}{c}\text { mejorar la actitud } \\
\text { personal frente al } \\
\text { riesgo. Disminuir el } \\
\text { riesgo } \\
\text { materializado y } \\
\text { fortalecer la } \\
\text { percepción global } \\
\text { de seguridad. }\end{array}$} & $\begin{array}{l}\text { 10. ( } \mathrm{N}^{\circ} \text { de pacientes que } \\
\text { clarifican las pautas de } \\
\text { prevención del riesgo } \\
\text { institucional / } \mathrm{N}^{\circ} \text { de } \\
\text { pacientes que son incluidos } \\
\text { en el programa de cuidado } \\
\text { seguro) } \times 100 \text {. }\end{array}$ & $\begin{array}{l}\text { 11. ( } \mathrm{N}^{\circ} \text { de cuidadores } \\
\text { familiares que clarifican } \\
\text { las pautas de } \\
\text { prevención del riesgo } \\
\text { institucional } / \mathrm{N}^{\circ} \text { de } \\
\text { cuidadores familiares } \\
\text { que son incluidos en el } \\
\text { programa de cuidado } \\
\text { seguro) } \times 100 \text {. }\end{array}$ & \\
\hline & & $\begin{array}{l}\text { 12. ( } \mathrm{N}^{\circ} \text { de pacientes que } \\
\text { mejoran la actitud personal } \\
\text { frente al riesgo institucional } \\
/ \mathrm{N}^{\circ} \text { de pacientes que son } \\
\text { incluidos en el programa de } \\
\text { cuidado seguro) } \times 100 .\end{array}$ & $\begin{array}{l}\text { 13. ( } \mathrm{N}^{\circ} \text { de cuidadores } \\
\text { familiares que mejoran } \\
\text { el nivel de actitud } \\
\text { personal frente al riesgo } \\
\text { institucional / } \mathrm{N}^{\circ} \text { de } \\
\text { cuidadores familiares } \\
\text { que son incluidos en el } \\
\text { programa de cuidado } \\
\text { seguro) } \times 100 \text {. }\end{array}$ & \\
\hline & & $\begin{array}{l}\text { 14. ( } \mathrm{N}^{\circ} \text { de pacientes que } \\
\text { mejoran el la percepción } \\
\text { global de seguridad } \\
\text { institucional } / \mathrm{N}^{\circ} \text { de } \\
\text { pacientes que son incluidos } \\
\text { en el programa de cuidado } \\
\text { seguro) } \times 100 \text {. }\end{array}$ & $\begin{array}{l}\text { 15. ( } \mathrm{N}^{\circ} \text { de cuidadores } \\
\text { familiares que mejoran } \\
\text { el la percepción global } \\
\text { de seguridad } \\
\text { institucional / } \mathrm{N}^{\circ} \text { de } \\
\text { cuidadores familiares } \\
\text { que son incluidos en el } \\
\text { programa de cuidado } \\
\text { seguro) } \times 100 \text {. }\end{array}$ & \\
\hline $\begin{array}{l}\text { Fortalecimiento } \\
\text { de la capacidad } \\
\text { de cuidar }\end{array}$ & $\begin{array}{l}\text { Mejorar la } \\
\text { capacidad de } \\
\text { cuidado de las } \\
\text { personas enfermas } \\
\text { y de sus } \\
\text { cuidadores } \\
\text { familiares. }\end{array}$ & $\begin{array}{l}\text { 16. ( } \mathrm{N}^{\circ} \text { de pacientes que } \\
\text { mejoran su nivel de } \\
\text { información sobre el } \\
\text { cuidado de su condición / } \\
\mathrm{N}^{\circ} \text { de pacientes que son } \\
\text { incluidos en el programa de } \\
\text { fortalecimiento de la } \\
\text { capacidad de cuidado) } \mathrm{x} \\
100 .\end{array}$ & $\begin{array}{l}\text { 17. ( } \mathrm{N}^{\circ} \text { de cuidadores } \\
\text { familiares que mejoran } \\
\text { su habilidad de cuidado } \\
/ \mathrm{N}^{\circ} \text { de cuidadores } \\
\text { familiares que son } \\
\text { incluidos en el programa } \\
\text { para el fortalecimiento } \\
\text { de la habilidad de } \\
\text { cuidado) } \times 100\end{array}$ & \\
\hline $\begin{array}{l}\text { Soporte social } \\
\text { con uso de TIC }\end{array}$ & $\begin{array}{c}\text { Mejorar la } \\
\text { percepción de } \\
\text { soporte social } \\
\text { mediante el uso } \\
\text { adecuado de las } \\
\text { TIC. }\end{array}$ & $\begin{array}{l}\text { 18. ( } \mathrm{N}^{\circ} \text { de pacientes que } \\
\text { mejoran su nivel de } \\
\text { percepción de soporte } \\
\text { social / } \mathrm{N}^{\circ} \text { de pacientes que } \\
\text { son incluidos en el } \\
\text { Programa de soporte social } \\
\text { con uso de las } \mathrm{TIC} \text { ) } \times 100 \text {. }\end{array}$ & $\begin{array}{l}\text { 19. }\left(\mathrm{N}^{\circ} \text { de cuidadores }\right. \\
\text { familiares que mejoran } \\
\text { su nivel de percepción } \\
\text { de soporte social / } \mathrm{N}^{\circ} \\
\text { de cuidadores familiares } \\
\text { que son incluidos en el } \\
\text { Programa de soporte } \\
\text { social con uso de las } \\
\mathrm{TIC}) \times 100 \text {. }\end{array}$ & \\
\hline
\end{tabular}

Fuente: Datos del estudio, 2012-2014. 
Tabla 2. Ejemplo de la hoja de vida de un indicador para la disminución de la carga del cuidado de una persona con enfermedad crónica.

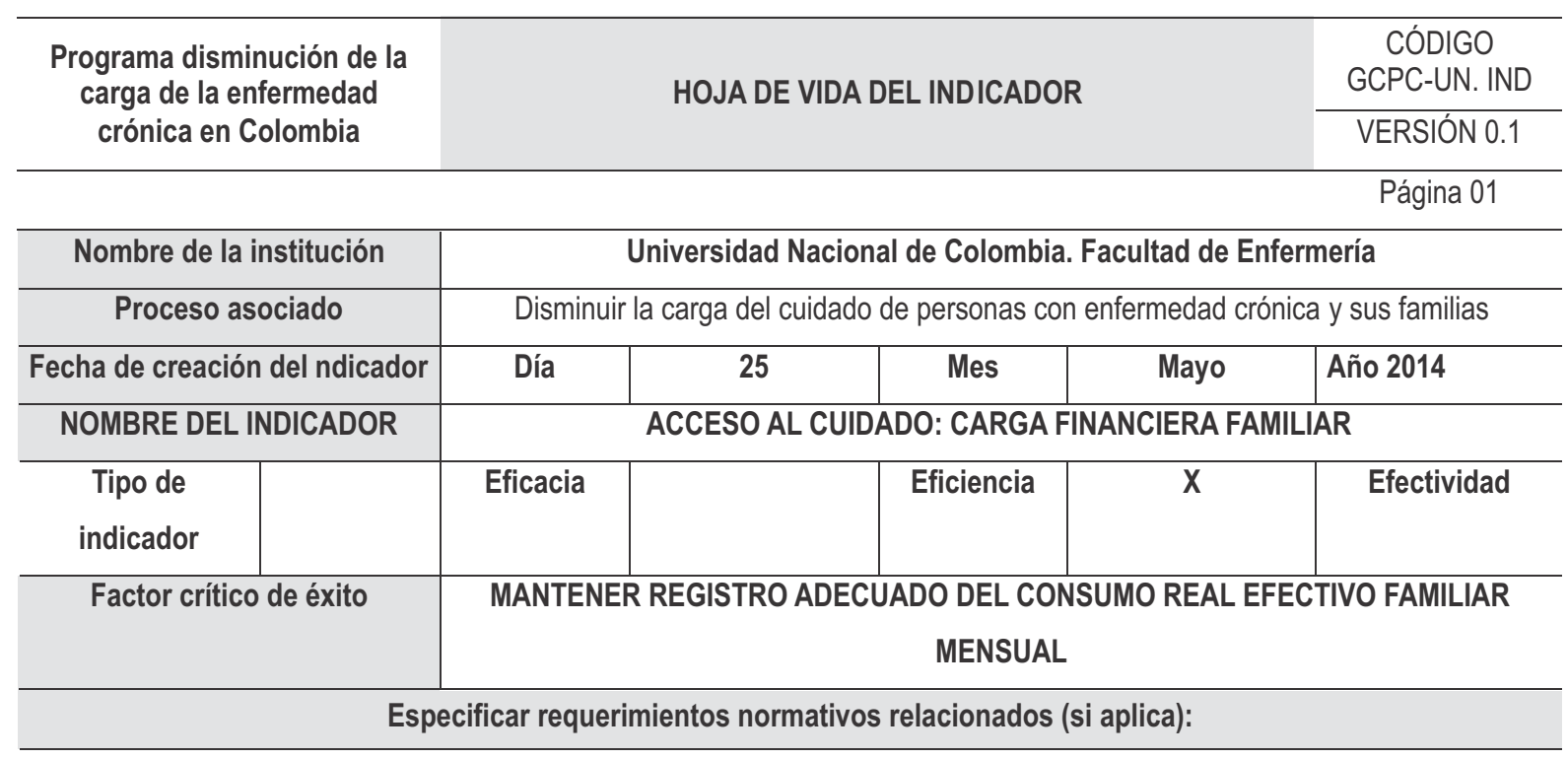

Ley 100 de 1993; Ley 1438 de 2011; Decreto 1011 de 2006; Resolución 1441 de 2013; Ley 1384 de 2010. (abril 19). Ley Sandra Ceballos; Ley 1733 de 2014 Consuelo Devis Saavedra.

\section{Descripción de las variables}

El Consumo real efectivo pondera las variables de alimentos, vivienda, transporte, salud y comunicaciones.

\section{Descripción del indicador}

El indicador retoma los datos de la encuesta "Costo financiero del cuidado de la enfermedad crónica" que permite determinar el consumo real efectivo familiar y determinar a través de este, cuál es el nivel de carga que dicho consumo genera. Para analizar la carga financiera se emplea la metodología CARACOL (Carga financiera atribuible al cuidado familiar de una persona con enfermedad crónica en Colombia).

\begin{tabular}{|c|c|c|c|c|c|c|}
\hline $\begin{array}{l}\text { Fórmula del } \\
\text { indicador }\end{array}$ & $\begin{array}{l}\text { Fuente de } \\
\text { datos }\end{array}$ & $\begin{array}{c}\text { Nivel de } \\
\text { aplicabilidad }\end{array}$ & $\begin{array}{l}\text { Precaución de } \\
\text { medición }\end{array}$ & $\begin{array}{l}\text { Unidad de } \\
\text { medida }\end{array}$ & Meta & $\begin{array}{l}\text { Responsable de } \\
\text { la medición }\end{array}$ \\
\hline $\begin{array}{c}\text { ( } \mathrm{N}^{\circ} \text { de familias } \\
\text { que logran } \\
\text { mantener o } \\
\text { disminuir la carga } \\
\text { financiera con el } \\
\text { cuidado de la } \\
\text { persona enferma / } \\
\mathrm{N}^{\circ} \text { de familias que } \\
\text { son incluidas en } \\
\text { el Programa de } \\
\text { Disminución de la } \\
\text { carga financiera } \\
\text { con el cuidado) x } \\
100 .\end{array}$ & $\begin{array}{l}\text { Registros y } \\
\text { recibos de } \\
\text { consumo } \\
\text { familiar. }\end{array}$ & $\begin{array}{c}\text { Grupo } \\
\text { familiar } \\
\text { mediante } \\
\text { entrevista a } \\
\text { alguno de los } \\
\text { integrantes } \\
\text { de la Diada. }\end{array}$ & $\begin{array}{l}\text { Los gastos deben } \\
\text { ser calculados por } \\
\text { mes en la moneda } \\
\text { local. }\end{array}$ & Porcentaje & $\begin{array}{l}\text { Mantenimiento del } \\
\text { consumo real } \\
\text { efectivo o } \\
\text { modificación con } \\
\text { disminución. }\end{array}$ & $\begin{array}{l}\text { Coordinadores del } \\
\text { Programa de } \\
\text { Disminución de la } \\
\text { carga financiera a } \\
\text { nivel institucional. }\end{array}$ \\
\hline \multicolumn{7}{|c|}{ Forma de obtención de la información } \\
\hline
\end{tabular}

Entrevista directa con cada familia en su sitio de vivienda o en un lugar de conveniencia del grupo familiar.

\section{Frecuencia de medición del indicador}

\section{Trimestral.}




\section{Cómo se interpreta}

La interpretación se fundamenta en la comparación de la carga anterior con la actual. Suponiendo que la EC cada vez genera más costo a la familia se asume que el mantener o disminuir la carga financiera familiar es deseable.

\begin{tabular}{|c|c|c|c|c|c|c|}
\hline \multicolumn{7}{|c|}{ Descripción de los rangos } \\
\hline Normal & & Riesgo & & Problema & & \\
\hline \multirow[t]{4}{*}{$\begin{array}{l}\text { Nivel de carga } \\
\text { actual }\end{array}$} & $\begin{array}{l}\text { Se aceptan } \\
\text { valores } \\
\text { iguales o } \\
\text { inferiores. }\end{array}$ & $\begin{array}{c}\text { Aumento de } \\
\text { la carga }\end{array}$ & $\begin{array}{l}\text { Se entre a revisar } \\
\text { qué ha sucedido y } \\
\text { en qué rubro del } \\
\text { consumo real } \\
\text { efectivo. }\end{array}$ & $\begin{array}{c}\text { Si la carga } \\
\text { sube más de } \\
25 \% .\end{array}$ & Acción correctiva & $\begin{array}{l}\text { Buscar apoyo y } \\
\text { remisión de la red } \\
\text { social disponible } \\
\text { para la familia. }\end{array}$ \\
\hline & \multicolumn{6}{|c|}{ Resultados: } \\
\hline & \multicolumn{6}{|c|}{ Período } \\
\hline & & 1 & 2 & 3 & 4 & 5 \\
\hline \multicolumn{7}{|c|}{ Variable 1} \\
\hline \multicolumn{7}{|c|}{ Variable 2} \\
\hline \multicolumn{7}{|c|}{ Resultado (aplicar fórmula) } \\
\hline \multicolumn{7}{|c|}{ Análisis de datos } \\
\hline Fecha de & dición & & & & & \\
\hline
\end{tabular}

Fuente: Datos de la investigación con base en matriz de indicadores, sistema de mejor gestión UN SIMEGE, 2014.

Tal como se ha señalado, cualquier reforma que se proponga dentro de un sistema de salud debe estar basada en una metodología de evaluación que sustituya la medición de variables por el empleo de indicadores de resultados. Si bien estos no proporcionan una visión exhaustiva de las condiciones de salud de toda la población, sí resultan indispensables para orientar con más eficacia los esfuerzos sectoriales encaminados a lograr el mayor impacto posible en los programas de salud pública (47).

Frente a la responsabilidad que genera conocer que la carga del cuidado es alta o excesiva en algunas de nuestras familias que viven la situación de cuidar a sus seres queridos con EC (48-50) la propuesta de indicadores para intervenir es más que necesaria. Por otro lado, responde a lo propuesto por la Organización Mundial de la Salud en su proyecto "Cuidados Innovadores para Condiciones Crónicas" que asume que en el cuidado es en donde se requiere el mayor énfasis actualmente"; de igual forma atiende el ejercicio a los lineamientos de la Organización Panamericana de Salud que basado en el Modelo de Cuidado Crónico sugiere que el mismo se complemente con la realidad de la asistencia (50). Bajo estos mismos lineamientos los indicadores reportados responden a consideraciones de un enfoque de cuidado que debe estar centrado en el paciente y su familia, así como al acceso, la propuesta de mejores prácticas y la evaluación de las estrategias de mejora de calidad, sin dejar de lado el fortalecimiento de la capacidad de cuidar.(50).

Si bien no existe una fórmula única para fortalecer el Sistema de Seguridad Social en Salud que es inmaduro según parámetros vigentes (4), la propuesta de indicadores lo respalda frente a su mayor reto con el cuidado de las personas con enfermedad crónica y el apoyo a sus familias que son quienes asumen la mayor parte de la responsabilidad(7-51) con consecuencias devastadoras.

\section{Conclusiones}

Los 19 indicadores establecidos permiten en conjunto medir la carga familiar del cuidado de la enfermedad crónica en el contexto colombiano y pueden ser aplicables al contexto de América Latina. Esta medición es necesaria e indispensable para conocer, entender, orientar y corregir la ejecución de las estrategias, acciones y resultados de la aplicación del Modelo para la disminución de la carga del cuidado de la enfermedad crónica en los aspectos referentes a las personas con enfermedad y sus familias.

Poder comunicar los resultados del cumplimiento de estrategias de acceso, seguridad y continuidad en el cuidado, así como las del fortalecimiento de la capacidad de cuidar de las personas con enfermedad y sus familias con soporte social con uso de TIC para saber cómo se cumplen los planteamientos teóricos conceptuales del MDCC-EC dando cumplimiento a los requisitos de los usuarios con el servicio, permite de una parte evaluar y de otra, proponer políticas públicas que permitan agregar valor al Sistema General de Seguridad Social cuando éste enfrenta el reto de la cronicidad como un aspecto prioritario de la salud pública. 


\section{Financiación}

Programa para la Disminución de la carga de la Enfermedad Crónica no Trasmisible en Colombia, con el apoyo financiero de COLCIENCIAS, código 501753730890

\section{Conflicto de intereses}

Las autoras declaran libremente no tener conflicto de intereses.

\section{Referencias}

1. Barrera L, Pinto N, Carrillo GM, Chaparro L, Sánchez B. Cuidando a los cuidadores: familiares de personas con enfermedad crónica. Bogotá: Empresa Editorial Universidad Nacional de Colombia, 2010.

2. Organización Mundial de la Salud. Estadísticas sanitarias Mundiales [Internet]. 2014. [Acceso 14 de febrero de 2015]. Disponible en: http://apps.who.int/iris/ bitstream/10665/112817/1/WHO_HIS_HSI_14.1_spa.p df?ua $=1 \& u a=1$

3. Organización Mundial de la Salud. Informe Sobre la Situación Mundial de las Enfermedades No Transmisibles. [Internet]. 2014. [Acceso 14 de febrero de 2015]. Disponible en: http://apps.who.int/iris/bitstream/ 10665/149296/1/WHO_NMH_NVI_15.1_spa.pdf?ua=1

4. Samb B, Desai N, Nishtar S, Mendis S, Bekeman H, Wright A. Prevention and management of chronic disease: a litmus test for health systems strengthening in low-income and middle-income countries. The Lancet. 2010; 376(9754): 1785-1797.

5. Agudelo CA, De la Hoz F, Mojica MJ, Eslava JC, Robledo M R, Cifuentes $P$, et al. Prioridades de Investigación en Salud en Colombia: Perspectiva de los Investigadores. Rev. Salud pública [revista en internet]. 2009 abril [Acceso 1 de junio de 2015]; 11(2):301-309. Disponible en: http://www.scielosp.org/scielo.php?script =sci_arttext\&pid=S0124-00642009000200014\&Ing=en. http://dx.doi.org/10.1590/S0124-00642009000200014.

6. World Health Organization. Innovative Care for Chronic Conditions [revista en internet]. 2002. [Acceso 14 de febrero de 2015]. Disponible en: http://www.who.int/chp/ knowledge/publications/icccglobalreport.pdf?ua=1

7. Robles L. El cuidado en el hogar a los enfermos crónicos: un sistema de auto atención. Cad. SaúdePública 2004; 20(2): 618-625.

8. Barrera L, PintoN, Sánchez B. Evaluación de un programa para fortalecer a los cuidadores familiares de enfermos crónicos. Rev. Salud pública. 2006; 8(2): 141152.

9. Breinbauer H, Vasquez H, Mayanz S, Guerra C, Millán K. Validación en Chile de la Escala de Sobrecarga del Cuidador de Zarit en sus versiones original y abreviada. Rev. méd. Chile. 2009: 137(5): 657-665.

10. Daivadanam M. Pathways to catastrophic health expenditure for acute coronary syndrome in Kerala:
'Good health at low cost'?. BMC Public Health. 2012; 12(1): 306.

11. Correa P. Out-Of-Pocket Health care Spending by the Chronically III in Chile. Procedia Economics and Finance. 2012: 88-97.

12. Piamjariyakul U, Yadrich DM, Russell C, Myer J, Prinyarux C, Vacek JL, et al. Patients' annual income adequacy, insurance premiums and out-of-pocket expenses related to heart failure care. Heart \& Lung: The Journal of Acute and Critical Care. 2014; 43(5): 469-475.

13. Mann BS, Barnieh L, Tang K, Campbell DJT, Clement F, et al. Association between Drug Insurance Cost Sharing Strategies and Outcomes in Patients with Chronic Diseases: A Systematic Review. 2014. PLoS ONE 9(3): e89168.

14. Chen A, Pollack C. Who Pays? Cost-Sharing, Tradeoffs, and the Physicians' Role in Decision Making. Journal of general internal medicine. 2012; 27(9): 1097-1099.

15. Luczak J, Garcia P. Financial burden of drug expenditures in Poland. Health Policy. 2012; 105 (2-3): 256-264.

16. Daniel W. Lai L. Effect of Financial Costs on Caregiving Burden of Family Caregivers of Older Adults, SAGE Open. 2012; 2(4): 1-14.

17. Fang K, Ma C, Jiang Y, Ye L, Shia B, Ma S. Illness, medical expenditure and household consumption: observations from Taiwan. BMC public health. 2013; 13(1): 743.

18. Mmopelwa G, Ngwenya BN, Sinha N, Sanders JB. Caregiver characteristics and economic cost of homebased care: a case study of Maun and Gumare villages in North West District, Botswana. Chronic IIIn. 2013; 9(1): 315.

19. Sado M, Inagaki A, Koreki A, Knapp M, Kissane LA, Mimura M, et al. The cost of schizophrenia in Japan. Neuropsychiatr Dis Treat. 2013; 9: 787-98.

20. Nishi A, McWilliams JM, Noguchi $H$, Hashimoto $H$, Tamiya N, Kawachi I.. Health benefits of reduced patient cost sharing in Japan.Bulletin of the World Health Organization. 2012; 90(6): 426-435.

21. Khang, Y. "Burden of No communicable Diseases and National Strategies to Control Them in Korea." Journal of Preventive Medicine and Public Health. 2013; 46(4): 155-164.

22. Li X, Shen JJ, Lu J, Wang Y, Sun M, Li C, et al. Household catastrophic medical expenses in eastern China: determinants and policy implications. BMC health services research. 2013; 13(1):506.

23. Fang K, Jiang Y, Shia B, Ma S. Impact of illness and medical expenditure on household consumptions: a survey in western China. PLoS One. 2012; 7(12):1-8.

24. Van Minh H, Tran BX. Assessing the household financial burden associated with the chronic non-communicable diseases in a rural district of Vietnam. Global health action. 2012; 5. Disponible en: http://www. globalhealthaction.net/index.php/gha/article/view/1889 2

25. Valtorta NK, Hanratty B. Socioeconomic variation in the financial consequences of ill health for older people with chronic diseases: A systematic review. Maturitas. Abril 2013; 74(4): 313-333. 
26. Essue BM, Wong G, Chapman J, Li Q, Jan S. How are patients managing with the costs of care for chronic kidney disease in Australia? A cross-sectional study. BMC nephrology. 2013; 14(1):5.

27. R Ramachandran, V Jha .Kidney transplantation is associated with catastrophic out of pocket expenditure in India.PLoS One. 2013; 8(7):1-8.

28. Arredondo A, Zuñiga A. Epidemiological changes and financial consequences of hypertension in Latin America: implications for the health system and patients in Mexico Cad. SaúdePública. Marzo 2012; 28(3): 497-502.

29. McRae I, Yen L, Jeon YH, Herath P, Essue B. Multimorbidity is associated with higher out-of-pocket spending: a study of older Australians with multiple chronic conditions. Aust J Prim Health. 2013; 19(2): 144-9.

30. Regidor, E. Los determinantes sociales de la salud, la posición socioeconómica y la inequidad en salud. Rev Panam Salud Pública. 2006; 20(4): 271-272.

31. Casas JA, Gwatkin DR. Las muchas dimensiones de la equidad en la salud. Revista Panamericana de Salud Pública. 2002; 11(5-6): 1-3.

32. Dantés HG, Castro V, Franco M, Bedregal P, García JR, Espinoza A, et al. La carga de la enfermedad en países de América Latina. Salud pública de méxico. 2011; 53 (supp 2): 72-77.

33. Velásquez A. Guía Metodológica para las Estimaciones Epidemiológicas del Estudio de Carga de Enfermedad. Lima: Promoviendo alianzas y estrategias. Abt Associates Inc. Noviembre de 2006.

34. Santos H. Los determinantes sociales, las desigualdades en salud y las políticas, como temas de investigación. Revista Cubana de Salud Pública. 2011; 37(2): 136-144.

35. Vargas LM. Marco para el cuidado de la salud en situaciones de enfermedad crónica. Investigación en Enfermería: Imagen y Desarrollo. 2011; 12(1): 79-94.

36. Corbin JM. The Corbin and Strauss Chronic Illness Trajectory model: an update. SchlnqNursPract. 1998; 12(1): 33-41.

37. Sánchez B, Carrillo GM, Barrera L, Chaparro L. Carga del cuidado de la enfermedad crónica no transmisible. Aquichan. 2013; 13(2): 247-260.

38. Hernández C, Orozco E, Arredondo A. Modelos conceptuales y paradigmas en salud pública. Revista de salud pública. 2012; 14(2): 315-324.

39. Coleman K, Austin BT, Brach C, Wagner EH. Evidence on the Chronic Care Model in the new millennium. Health affairs. 2009; 28(1): 75-85.

40. Universidad Nacional de Colombia. Manual De Indicadores, Sistema De Mejor Gestión UN SIMEGE. [revista en internet]. 2014. [Acceso 15 de Septiembre de 2014] Disponible en: http://www.agronomia.unal.edu.co /home/files/Guia_indicadores.pdf

41. República de Colombia. Ministerio de Salud. (04 de Octubre de 1993). Resolución 008430 de 1993 Por la cual se establecen las normas científicas, técnicas y administrativas para la investigación en salud. Bogotá.

42. Consejo de organizaciones internacionales de las ciencias médicas. Pautas éticas internacionales para la investigación biomédica en seres humanos preparadas por el consejo de organizaciones internacionales de las ciencias médicas (ClOMS). Ginebra: 2012.

43. Jiménez RE. Indicadores de calidad y eficiencia de los servicios hospitalarios: una mirada actual. Revista cubana de salud pública. 2004; 30(1): 17-36.

44. Hernández A. Calidad de vida y medio ambiente urbano: indicadores locales de sostenibilidad y calidad de vida urbana. Revista INVI. 2009; 24(65): 79-111.

45. Gabriel C S, da Costa MRA, Rocha FLR, Bernardesm A, Miguelaci T, Silva MD. Utilización de indicadores de desempeño en servicio de enfermería de hospital público. Rev. latinoam. enferm., Ribeirão Preto. 2011. 19(5): 12471256.

46. Mirón JA, Alonso M. Medidas de frecuencia, asociación e impacto en investigación aplicada. Medicina y Seguridad del Trabajo. 2008; 54(211): 93-102.

47. De la Fuente JR, Tapia Conyer. La medición en salud a través de indicadores. Revista Panamericana de Salud Pública. 2003; 13 (1): 56-58.

48. Carrillo GM, Chaparro L, Sánchez B. Carga del cuidado en cuidadores familiares de personas con enfermedad crónica en la región pacífica colombiana. Ciencia y enfermería. 2014; 20(2): 83-91.

49. Barrera L, Sánchez B, Carrillo GM. La carga del cuidado en cuidadores de niños con enfermedad crónica. Revista Cubana de Enfermería. 2013; 29(1): 39-47.

50. Organização Mundial da Saúde. Cuidados inovadores para condições crônicas: componentes estruturais de ação: relatório mundial. 2003.

51. Barrera L, Pinto N, Sánchez B. Evaluación de un programa para fortalecer a los cuidadores familiares de enfermos crónicos. Rev Salud Pública. 2006; 8(2): 141-152. 